\title{
Prolongability of Ordinary Differential Equations
}

Yoshishige Haraoka

To cite this article: Yoshishige Haraoka (2013) Prolongability of Ordinary Differential Equations, Journal of Nonlinear Mathematical Physics 20: Supplement 1, 70-84, DOI: https://doi.org/10.1080/14029251.2013.862435

To link to this article: https://doi.org/10.1080/14029251.2013.862435

Published online: 04 January 2021 


\title{
Prolongability of Ordinary Differential Equations
}

\author{
Yoshishige Haraoka \\ Department of Mathematics, Kumamoto University \\ Kumamoto 860-8555, Japan \\ haraoka@kumamoto-u.ac.jp \\ Received 31 August 2012 \\ Accepted 28 May 2013
}

\begin{abstract}
We extend the notion of deformation to inverse operations of restrictions of completely integrable systems to regular or singular locus, and call the extended notion prolongation. We show that a prolongability determines uniquely a Fuchsian ordinary differential equation of rank three with three regular singular points. This seems similar to that the deformation equation determines the accessory parameters as a function of the geometric moduli. Relations between prolongations and middle convolutions is also studied.
\end{abstract}

Keywords: Accessory parameter, Pfaffian system, middle convolution, restriction, index of rigidity.

2010 Mathematics Subject Classification: 32G34, 58A17

\section{Introduction}

Consider a system of Fuchsian ordinary differential equations

$$
\frac{d Y}{d x}=A(x, t) Y,
$$

where $t=\left(t_{1}, t_{2}, \ldots, t_{p}\right)$ denotes the set of regular singular points. The deformation equation of (1.1) is the complete integrability condition of the system of partial differential equations

$$
\left\{\begin{array}{l}
\frac{\partial Y}{\partial x}=A(x, t) Y, \\
\frac{\partial Y}{\partial t_{i}}=B_{i}(x, t) Y, \quad(1 \leq i \leq p),
\end{array}\right.
$$

where each $B_{i}(x, t)$ is rational in $x$. Thus to solve a deformation equation implies to construct a completely integrable system (1.2) which is a prolongation of the ordinary differential equation (1.1). Here we have called (1.2) a prolongation of (1.1) because the ordinary differential equation (1.1) is obtained as a restriction of (1.2) to the locus $t_{i}=$ const. for any $i$.

For some completely integrable system $\mathscr{M}$, we may take a restriction to a singular locus, and then obtain an ordinary differential equation $\mathscr{L}$. In this case, we also regard $\mathscr{M}$ as a prolongation of $\mathscr{L}$, and say that $\mathscr{L}$ is prolongable to $\mathscr{M}$. Thus prolongation is an extended notion of deformation.

Since we can send three singular points, say $t_{1}, t_{2}, t_{3}$, of $(1.1)$ to $0,1, \infty$ by an automorphism of $\mathbb{P}^{1}$, the essential variables of the system (1.2) are $x$ and $t_{4}, \ldots, t_{p}$. Then we cannot deform the ordinary differential equation (1.1) if $p \leq 3$. On the other hand, even in the case $p=3$, the ordinary 
differential equation may have a prolongation in the sense of the inverse of a restriction to a singular locus.

In this paper, we study a Fuchsian ordinary differential equation of rank three with three singular points and two accessory parameters, and show that the special values of the accessory parameters are determined by a prolongability. $\S 3$ is devoted to this consideration. This result looks similar to that the deformation equation determines the accessory parameters as functions of the positions of the singular points.

The addition and the middle convolution are introduced by Katz [7], [1], [2], and are basic operations for Fuchsian ordinary differential equations. We have shown that these operations leaves the deformation equation invariant [5]. Then we are interested in the relation between the prolongation and these operations. We study this problem in $\S 4$.

\section{Dotsenko-Fateev Differential Equation}

In studying the conformal field theory, Dotsenko and Fateev [3] obtained a differential equation satisfied by the Selberg type integral

$$
u(x)=\int_{\Delta} s_{1}^{a}\left(s_{1}-1\right)^{b}\left(s_{1}-x\right)^{c} s_{2}{ }^{a}\left(s_{2}-1\right)^{b}\left(s_{2}-x\right)^{c}\left(s_{1}-s_{2}\right)^{g} \frac{d s_{1} \wedge d s_{2}}{s_{1} s_{2}} .
$$

It is a third order Fuchsian ordinary differential equation

$$
x^{2}(x-1)^{2} u^{\prime \prime \prime}+p(x) u^{\prime \prime}+q(x) u^{\prime}+r(x) u=0,
$$

where $p(x), q(x), r(x)$ are polynomials of degrees $3,2,1$, respectively, and the Riemann scheme of (2.2) is given by

$$
\left\{\begin{array}{ccc}
x=0 & x=1 & x=\infty \\
0 & 0 & -2 c \\
a+c & b+c+1 & -(a+b+2 c+g) \\
2 a+2 c+g & 2 b+2 c+g+2 & -(2 a+2 b+2 c+g)
\end{array}\right\} .
$$

If we write $r(x)=r_{1} x+r_{0}$, the polynomials $p(x), q(x)$ and the coefficient $r_{1}$ are uniquely determined by the Riemann scheme (2.3), while $r_{0}$ is not. Thus $r_{0}$ is the accessory parameter of the equation (2.2), and in the equation (2.2) it takes the special value

$$
r_{0}=c(2 a+2 c+g-1)(2 a+2 b+2 c+g) .
$$

Since the differential equation (2.2) is uniquely determined by the solution (2.1), we can understand that this special value of the accessory parameter $r_{0}$ is so chosen that the differential equation (2.2) with Riemann scheme (2.3) admits the integral (2.1) as a solution.

The equation (2.2) is transformed into a Fuchsian system

$$
\frac{d U}{d x}=\left(\frac{A_{1}}{x}+\frac{A_{2}}{x-1}\right) U
$$

where

$$
A_{1}=\left(\begin{array}{ccc}
2 a+2 c+g & 0 & b \\
0 & 0 & 0 \\
0 & 2 b+g & a+c
\end{array}\right), A_{2}=\left(\begin{array}{ccc}
0 & 0 & 0 \\
0 & 2 b+2 c+g & a \\
2 a+g & 0 & b+c
\end{array}\right)
$$


The unknown vector $U$ is given by

$$
U(x)=\left(\begin{array}{c}
\int_{\Delta} \Phi \eta_{1} \\
\int_{\Delta} \Phi \eta_{2} \\
\int_{\Delta} \Phi \eta_{3}
\end{array}\right)
$$

where we set

$$
\begin{aligned}
& \Phi={s_{1}}^{a}\left(s_{1}-1\right)^{b}\left(s_{1}-x\right)^{c} s_{2}^{a}\left(s_{2}-1\right)^{b}\left(s_{2}-x\right)^{c}\left(s_{1}-s_{2}\right)^{g} \\
& \eta_{1}=\frac{d s_{1} \wedge d s_{2}}{s_{1} s_{2}}, \eta_{2}=\frac{d s_{1} \wedge d s_{2}}{\left(s_{1}-1\right)\left(s_{2}-1\right)}, \eta_{3}=\frac{d s_{1} \wedge d s_{2}}{s_{1}\left(s_{2}-1\right)}+\frac{d s_{1} \wedge d s_{2}}{\left(s_{1}-1\right) s_{2}} .
\end{aligned}
$$

The first element of the vector $U(x)$ coincides with $u(x)$ in (2.1).

Kato [6] found the same system (2.4) as a restriction of the system satisfied by Appell's hypergeometric function $F_{4}$ to a singular locus. First he obtained a Pfaffian system with a logarithmic form

$$
d V=\left[P_{1} \frac{d x}{x}+P_{2} \frac{d y}{y}+P_{3} \frac{d x}{x-1}+P_{4} \frac{d y}{y-1}+P_{5} \frac{d(x-y)}{x-y}\right] V
$$

from the system of differential equations satisfied by $F_{4}\left(\alpha, \beta, \gamma, \gamma^{\prime} ; x, y\right)$, where

$$
\begin{aligned}
P_{1} & =\left(\begin{array}{cccc}
0 & 1 & 0 & 0 \\
0 & 1-\gamma & 0 & 0 \\
0 & \varepsilon & 0 & 1 \\
0 & 0 & 0 & 1-\gamma
\end{array}\right), \quad P_{2}=\left(\begin{array}{cccc}
0 & 0 & 1 & 0 \\
0 & 0 & \varepsilon & 1 \\
0 & 0 & 1-\gamma & 0 \\
0 & 0 & 0 & 1-\gamma
\end{array}\right), \\
P_{3} & =\left(\begin{array}{cccc}
0 & 0 & 0 & 0 \\
-\alpha \beta & -\gamma^{\prime} & \varepsilon & 0 \\
0 & 0 & 0 & 0 \\
0 & 0 & -(\alpha+\varepsilon)(\beta+\varepsilon) & -\gamma^{\prime}
\end{array}\right) \\
P_{4} & =\left(\begin{array}{cccc}
0 & 0 & 0 & 0 \\
0 & 0 & 0 & 0 \\
-\alpha \beta & \varepsilon & -\gamma^{\prime} & 0 \\
0 & -(\alpha+\varepsilon)(\beta+\varepsilon) & 0 & -\gamma^{\prime}
\end{array}\right) \\
P_{5} & =\left(\begin{array}{cccc}
0 & 0 & 0 & 0 \\
0 & \varepsilon & -\varepsilon & 0 \\
0 & -\varepsilon & \varepsilon & 0 \\
0 & 0 & 0 & 0
\end{array}\right)
\end{aligned}
$$

and

$$
\varepsilon:=\gamma+\gamma^{\prime}-\alpha-\beta-1
$$

By using the Pfaffian system (2.5), we can calculate the restriction to the singular locus $x=y$ as follows. Take a constant gauge transformation $V(x) \mapsto Q V(x)$ so that the residue matrix at $x=y$ 
becomes the diagonal matrix $\operatorname{diag}[0,0,0,-2 \varepsilon]$. Then, if we set

$$
Q\left(P_{1}+P_{2}\right) Q^{-1}=\left(\begin{array}{c|c}
C_{1} & * \\
\hline * & *
\end{array}\right), \quad Q\left(P_{3}+P_{4}\right) Q^{-1}=\left(\begin{array}{c|c}
C_{2} & * \\
\hline * & *
\end{array}\right),
$$

where we have partitioned the matrices in the right hand sides into $(3,1) \times(3,1)$-blocks, the restriction is given by the system

$$
\frac{d U}{d x}=\left(\frac{C_{1}}{x}+\frac{C_{2}}{x-1}\right) U
$$

Explicitly we have

$$
C_{1}=\left(\begin{array}{lcc}
0 & 2 & 0 \\
0 & \gamma^{\prime}-\alpha-\beta & 1 \\
0 & 0 & 2(1-\gamma)
\end{array}\right), C_{2}=\left(\begin{array}{ccc}
0 & 0 & 0 \\
-\alpha \beta & \gamma-\alpha-\beta-1 & 0 \\
0 & -2(\varepsilon+\alpha)(\varepsilon+\beta) & -2 \gamma^{\prime}
\end{array}\right) .
$$

If we set

$$
\begin{aligned}
& \alpha=-c, \quad \beta=-a-b-2-\frac{g}{2}, \\
& \gamma=-b-c-\frac{g}{2}, \quad \gamma^{\prime}=1-a-c-\frac{g}{2},
\end{aligned}
$$

we see

$$
\left(C_{1}, C_{2}\right)=\left(P^{-1} A_{1} P, P^{-1} A_{2} P\right)
$$

with some $P \in \mathrm{GL}(3, \mathbb{C})$. This implies that the restriction of the Pfaffian system (2.5) to the singular locus coincides with the Dotsenko-Fateev system (2.4). In other words, the Dotsenko-Fateev system (2.4) is prolongable into a completely integrable system in two variables.

\section{Prolongability}

In this section we consider the prolongability of Fuchsian systems of rank three with three singular points of spectral types $(1,1,1)$. First we describe the moduli space of such systems.

For a set $\alpha=\left\{\alpha_{1}, \alpha_{2}, \alpha_{3}\right\}$ of three distinct complex numbers, we set

$$
\mathscr{O}(\alpha)=\left\{B \in \mathfrak{g l}(3, \mathbb{C}) ; B \sim \operatorname{diag}\left[\alpha_{1}, \alpha_{2}, \alpha_{3}\right]\right\} .
$$

Let $\alpha=\left\{\alpha_{1}, \alpha_{2}, \alpha_{3}\right\}, \beta=\left\{\beta_{1}, \beta_{2}, \beta_{3}\right\}, \gamma=\left\{\gamma_{1}, \gamma_{2}, \gamma_{3}\right\}$ be sets of three distinct complex numbers satisfying

$$
|\alpha|+|\beta|+|\gamma|=0,
$$

where $|\alpha|:=\alpha_{1}+\alpha_{2}+\alpha_{3}$. We consider the moduli space

$$
\mathscr{M}_{\alpha, \beta, \gamma}=\left\{\left(B_{1}, B_{2}\right) \in \mathscr{O}(\alpha) \times \mathscr{O}(\beta) ;-\left(B_{1}+B_{2}\right) \in \mathscr{O}(\gamma)\right\} / \approx,
$$

where the equivalence relation is defined by

$$
\left(B_{1}, B_{2}\right) \approx\left(P B_{1} P^{-1}, P B_{2} P^{-1}\right) \quad(\exists P \in \mathrm{GL}(3, \mathbb{C})) .
$$


An element $\left[\left(B_{1}, B_{2}\right)\right] \in \mathscr{M}_{\alpha, \beta, \gamma}$ is called irreducible if $B_{1}$ and $B_{2}$ have no common invariant subspace except $\{0\}$ and $\mathbb{C}^{3}$. We denote by $\mathscr{M}_{\alpha, \beta, \gamma}^{\text {irr }}$ the subset of $\mathscr{M}_{\alpha, \beta, \gamma}$ of irreducible elements. With a representative $\left(B_{1}, B_{2}\right)$ of an element in $\mathscr{M}_{\alpha, \beta, \gamma}$, we associate a system of differential equations

$$
\frac{d U}{d x}=\left(\frac{B_{1}}{x}+\frac{B_{2}}{x-1}\right) U
$$

The equivalence relation (3.2) corresponds to the constant gauge transformation $U(x) \mapsto P U(x)$, and $\alpha, \beta, \gamma$ are the sets of local exponents of the system (3.3) at $x=0,1, \infty$, respectively. The accessory parameters of the system (3.3) is the parameters of the moduli space $\mathscr{M}_{\alpha, \beta, \gamma}$.

We can easily show the following.

Lemma 3.1. Let $\alpha=\left\{\alpha_{1}, \alpha_{2}, \alpha_{3}\right\}, \beta=\left\{\beta_{1}, \beta_{2}, \beta_{3}\right\}, \gamma=\left\{\gamma_{1}, \gamma_{2}, \gamma_{3}\right\}$ be any sets of three distinct complex numbers satisfying (3.1). For any element of $\mathscr{M}_{\alpha, \beta, \gamma}$, we can take a representative $\left(B_{1}, B_{2}\right)$ of the form

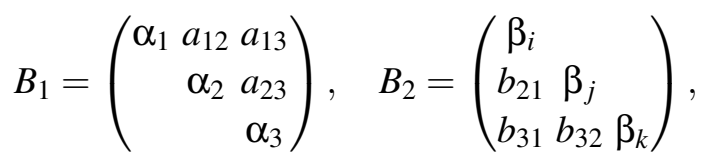

where $(i, j, k)$ is a permutation of $(1,2,3)$. Moreover, if $a_{12} a_{23} \neq 0$, we can take

$$
a_{12}=a_{23}=1,
$$

and in this case $a_{13}, b_{21}, b_{31}, b_{32}$ satisfy

$$
\begin{aligned}
& b_{32}+a_{13} b_{31}+b_{21}=\left(\alpha_{1}+\beta_{i}\right)\left(\alpha_{2}+\beta_{j}\right)+\left(\alpha_{2}+\beta_{j}\right)\left(\alpha_{3}+\beta_{k}\right) \\
& \quad+\left(\alpha_{3}+\beta_{k}\right)\left(\alpha_{1}+\beta_{i}\right)-\gamma_{1} \gamma_{2}-\gamma_{2} \gamma_{3}-\gamma_{3} \gamma_{1}, \\
& \left(\alpha_{1}+\beta_{i}\right) b_{32}+\left(\alpha_{2}+\beta_{j}\right) a_{13} b_{31}+\left(\alpha_{3}+\beta_{k}\right) b_{21}-b_{31}-a_{13} b_{32} b_{21} \\
& \quad=\left(\alpha_{1}+\beta_{i}\right)\left(\alpha_{2}+\beta_{j}\right)\left(\alpha_{3}+\beta_{k}\right)+\gamma_{1} \gamma_{2} \gamma_{3} .
\end{aligned}
$$

We see that, when $a_{12} a_{23} \neq 0$, we can take $a_{13}$ and $b_{32}$ as the accessory parameters.

We consider the Dotsenko-Fateev system (2.4). If we set

$$
\begin{aligned}
& \alpha^{0}=\{0, a+c, 2 a+2 c+g\}, \\
& \beta^{0}=\{0, b+c, 2 b+2 c+g\}, \\
& \gamma^{0}=\{-2 c,-(a+b+2 c+g),-(2 a+2 b+2 c+g)\},
\end{aligned}
$$

we have

$$
\left[\left(A_{1}, A_{2}\right)\right] \in \mathscr{M}_{\alpha^{0}, \beta^{0}, \gamma^{0}}
$$

We find a representative $\left(A_{1}^{0}, A_{2}^{0}\right)$ of $\left[\left(A_{1}, A_{2}\right)\right]$ of the form (3.4) given by

$$
\begin{aligned}
A_{1}^{0} & =\left(\begin{array}{lll}
0 & 1 & 0 \\
0 & a+c & 1 \\
0 & 0 & 2 a+2 c+g
\end{array}\right), \\
A_{2}^{0} & =\left(\begin{array}{ccc}
0 & 0 & 0 \\
-c(2 a+2 b+2 c+g) & b+c & 0 \\
0 & -(a+b+c+g)(2 c+g) & 2 b+2 c+g
\end{array}\right) .
\end{aligned}
$$


Thus in this case, the accessory parameters take the special values

$$
a_{13}=0, \quad b_{32}=-(a+b+c+g)(2 c+g) .
$$

Next, we study the prolongability. Since the inverse of a restriction to a singular locus is not unique, we must fix the rank and the singular locus of the result of prolongation in order to formulate the problem. Taking the facts explained in the last section into consideration, we take completely integrable systems of rank four on $\mathbb{P}^{2}$ with singular locus

$$
\{x=0\} \cup\{y=0\} \cup\{x=1\} \cup\{y=1\} \cup\{x=y\} \cup L_{\infty},
$$

where $L_{\infty}$ denotes the line at infinity. Then we can show that the prolongability to such completely integrable systems uniquely determines an element of the moduli space $\mathscr{M}_{\alpha, \beta, \gamma}$.

For $\alpha=\left\{\alpha_{1}, \alpha_{2}, \alpha_{3}\right\}$ and $\rho \in \mathbb{C}$, we set $\alpha-\rho 1=\left\{\alpha_{1}-\rho, \alpha_{2}-\rho, \alpha_{3}-\rho\right\}$.

Theorem 3.1. Let $\alpha, \beta, \gamma$ be sets of three distinct complex numbers satisfying

$$
|\alpha|+|\beta|+|\gamma|=0
$$

Suppose that there exists an irreducible and completely integrable Pfaffian system

$$
d V=\left[R_{1} \frac{d x}{x}+R_{2} \frac{d y}{y}+R_{3} \frac{d x}{x-1}+R_{4} \frac{d y}{y-1}+R_{5} \frac{d(x-y)}{x-y}\right] V
$$

of rank four with

$$
R_{5}=\operatorname{diag}\left[0,0,0, w_{5}\right]
$$

for some $w_{5} \in \mathbb{C}$, such that the restriction to the singular locus $x=y$ gives the system

$$
\frac{d U}{d x}=\left(\frac{B_{1}}{x}+\frac{B_{2}}{x-1}\right) U
$$

with $\left[\left(B_{1}, B_{2}\right)\right] \in \mathscr{M}_{\alpha, \beta, \gamma}^{i r}$.

Then there exist $\rho_{1}, \rho_{2} \in \mathbb{C}$ such that

$$
\alpha-\rho_{1} \mathbf{1}=\alpha^{0}, \beta-\rho_{2} \mathbf{1}=\beta^{0}, \gamma+\left(\rho_{1}+\rho_{2}\right) \mathbf{1}=\gamma^{0},
$$

where $\alpha^{0}, \beta^{0}, \gamma^{0}$ are given in (3.5) for some $(a, b, c, g) \in \mathbb{C}^{4}$, and we have

$$
\left(B_{1}-\rho_{1} I_{3}, B_{2}-\rho_{2} I_{3}\right) \in\left[\left(A_{1}^{0}, A_{2}^{0}\right)\right],
$$

where $A_{1}^{0}, A_{2}^{0}$ are given in (3.6). Moreover the Dotsenko-Fateev system (2.4) for generic parameters $(a, b, c, g)$ can be obtained in this way. 
Proof. We partition each $R_{j}$ into $(3,1) \times(3,1)$-blocks, and set

$$
R_{j}=\left(\begin{array}{c|c}
S_{j} & u_{j} \\
\hline v_{j} & w_{j}
\end{array}\right) .
$$

Then the relations among the $R_{j}$ 's and $B_{j}$ 's are given by

$$
S_{1}+S_{2}=B_{1}, \quad S_{3}+S_{4}=B_{2}
$$

The integrability condition of the system (3.7) is given by the relations

$$
\begin{aligned}
& {\left[R_{1}, R_{4}\right]=0, \quad\left[R_{2}, R_{3}\right]=0,} \\
& {\left[R_{1}, R_{2}+R_{5}\right]=0, \quad\left[R_{2}, R_{1}+R_{5}\right]=0,} \\
& {\left[R_{3}, R_{4}+R_{5}\right]=0, \quad\left[R_{4}, R_{3}+R_{5}\right]=0 .}
\end{aligned}
$$

We note that a gauge transformation $V(x, y) \mapsto x^{\lambda_{1}} y^{\lambda_{2}}(x-1)^{\lambda_{3}}(y-1)^{\lambda_{4}} V(x, y)$ causes a scalar shift $R_{j} \mapsto R_{j}+\lambda_{j}(1 \leq j \leq 4)$ to the system (3.7) and $B_{1} \mapsto B_{1}+\left(\lambda_{1}+\lambda_{2}\right), B_{2} \mapsto B_{2}+\left(\lambda_{3}+\lambda_{4}\right)$ to the system (3.8), and does not change the irreducibility and integrability. A constant gauge transformation by the matrix

$$
\left(\frac{Q}{1}\right) \in \mathrm{GL}(3, \mathbb{C}) \times \mathrm{GL}(1, \mathbb{C})
$$

to the system (3.7) also keeps the irreducibility and integrability, and sends $\left(B_{1}, B_{2}\right)$ to $\left(Q B_{1} Q^{-1}, Q B_{2} Q^{-1}\right)$. Thus, by operating these transformations, we may assume $\left(B_{1}, B_{2}\right)$ is of the normal form (3.4) with $(i, j, k)=(1,2,3)$ and $\alpha_{1}=\beta_{1}=0$, and (1,1)-entries of $S_{1}, S_{2}, S_{3}$ and $S_{4}$ are 0 . Then we can set

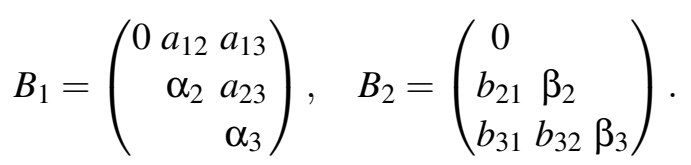

Rewriting the integrability condition (3.11) in terms of $S_{j}, u_{j}, v_{j}, w_{j}$, we get

$$
\begin{aligned}
& u_{2}=-u_{1}, v_{2}=-v_{1}, u_{4}=-u_{3}, v_{4}=-v_{3}, \\
& {\left[S_{1}, S_{2}\right]=0,\left[S_{3}, S_{4}\right]=0,} \\
& \left(S_{1}+S_{2}\right) u_{1}=\left(w_{1}+w_{2}+w_{5}\right) u_{1},\left(S_{3}+S_{4}\right) u_{3}=\left(w_{3}+w_{4}+w_{5}\right) u_{3}, \\
& v_{1}\left(S_{1}+S_{2}\right)=\left(w_{1}+w_{2}+w_{5}\right) v_{1}, v_{3}\left(S_{3}+S_{4}\right)=\left(w_{3}+w_{4}+w_{5}\right) v_{1}, \\
& {\left[S_{1}, S_{4}\right]=\left[S_{2}, S_{3}\right]=u_{1} v_{3}-u_{3} v_{1},} \\
& S_{1} u_{3}+S_{4} u_{1}=w_{1} u_{3}+w_{4} u_{1}, S_{2} u_{3}+S_{3} u_{1}=w_{2} u_{3}+w_{3} u_{1}, \\
& v_{3} S_{1}+v_{1} S_{4}=w_{1} v_{3}+w_{4} v_{1}, v_{3} S_{2}+v_{1} S_{3}=w_{2} v_{e}+w_{3} v_{1}, \\
& v_{1} u_{3}=v_{3} u_{1} .
\end{aligned}
$$


By using (3.10), we obtain

$$
\begin{aligned}
& B_{1} u_{1}=\left(w_{1}+w_{2}+w_{5}\right) u_{1}, v_{1} B_{1}=\left(w_{1}+w_{2}+w_{5}\right) v_{1}, \\
& B_{2} u_{3}=\left(w_{3}+w_{4}+w_{5}\right) u_{3}, v_{3} B_{2}=\left(w_{3}+w_{4}+w_{5}\right) v_{3}, \\
& B_{1} u_{3}+B_{2} u_{1}=\left(w_{1}+w_{2}\right) u_{3}+\left(w_{3}+w_{4}\right) u_{1}, \\
& v_{3} B_{1}+v_{1} B_{2}=\left(w_{1}+w_{2}\right) v_{3}+\left(w_{3}+w_{4}\right) v_{1}
\end{aligned}
$$

from (3.12). Suppose $u_{1}=0$. In this case we have $u_{3} \neq 0$, for otherwise the system (3.7) clearly becomes reducible. Now, by using (3.13) and $u_{1}=0$, we get

$$
B_{1} u_{3} \in\left\langle u_{3}\right\rangle, \quad B_{2} u_{3} \in\left\langle u_{3}\right\rangle,
$$

which implies $\left(B_{1}, B_{2}\right)$ is reducible. Hence we have $u_{1} \neq 0$. Similarly we see that $u_{3} \neq 0, v_{1} \neq 0$ and $v_{3} \neq 0$. Thus $w_{1}+w_{2}+w_{5}$ is an eigenvalue of $B_{1}$ with column eigenvector $u_{1}$ and row eigenvector $v_{1}$, and $w_{3}+w_{4}+w_{5}$ is an eigenvalue of $B_{2}$ with column eigenvector $u_{3}$ and row eigenvector $v_{3}$. In a similar argument, we can show that $u_{1}$ and $u_{3}$ are linearly independent.

Combining (3.10) and (3.12), we also get

$$
\begin{aligned}
& {\left[S_{1}, B_{1}\right]=0, \quad\left[B_{2}, S_{4}\right]=0,} \\
& {\left[S_{1}, B_{2}\right]+\left[B_{1}, S_{4}\right]=\left[B_{1}, B_{2}\right] .}
\end{aligned}
$$

By solving these relations, we have the relations

$$
S_{1}=c B_{1}, \quad S_{4}=(1-c) B_{2}
$$

for some $c \in \mathbb{C}$, and hence get

$$
S_{2}=(1-c) B_{1}, \quad S_{3}=c B_{2}
$$

Now we have

$$
\begin{aligned}
S_{1} u_{3}+S_{4} u_{1} & =c B_{1} u_{3}+(1-c) B_{2} u_{1} \\
& =c\left(B_{1} u_{3}+B_{2} u_{1}\right)+(1-2 c) B_{2} u_{1} .
\end{aligned}
$$

Thanks to (3.12) and (3.13), the left hand side and the first term in the right hand side are contained in $\left\langle u_{1}, u_{3}\right\rangle$, and hence

$$
(1-2 c) B_{2} u_{1} \in\left\langle u_{1}, u_{3}\right\rangle
$$

If $1-2 c \neq 0$, we have $B_{2} u_{1} \in\left\langle u_{1}, u_{3}\right\rangle$, which implies $B_{1} u_{3} \in\left\langle u_{1}, u_{3}\right\rangle$ by (3.13). Since $B_{1} u_{1} \in\left\langle u_{1}\right\rangle$ and $B_{2} u_{3} \in\left\langle u_{3}\right\rangle$ by (3.13), we have

$$
B_{1}\left\langle u_{1}, u_{3}\right\rangle \subset\left\langle u_{1}, u_{3}\right\rangle, \quad B_{2}\left\langle u_{1}, u_{3}\right\rangle \subset\left\langle u_{1}, u_{3}\right\rangle,
$$

which shows $\left(B_{1}, B_{2}\right)$ is reducible. Thus we have $c=1 / 2$, and

$$
S_{1}=S_{2}=\frac{1}{2} B_{1}, \quad S_{3}=S_{4}=\frac{1}{2} B_{2} .
$$

By using these relations, we have

$$
S_{1} u_{3}+S_{4} u_{1}=\frac{1}{2}\left(B_{1} u_{3}+B_{2} u_{1}\right)=\frac{1}{2}\left(\left(w_{1}+w_{2}\right) u_{3}+\left(w_{3}+w_{4}\right) u_{1}\right) .
$$


Since the left hand side coincides with $w_{1} u_{3}+w_{4} u_{1}$ by (3.12) and $u_{1}$ and $u_{3}$ are linearly independent, we have

$$
w_{1}=\frac{1}{2}\left(w_{1}+w_{2}\right), \quad w_{4}=\frac{1}{2}\left(w_{3}+w_{4}\right) .
$$

Thus we get

$$
w_{1}=w_{2}, \quad w_{3}=w_{4}
$$

By virtue of these relations, the integrability condition (3.12) can be rewitten as

$$
\begin{aligned}
& B_{1} u_{1}=\left(2 w_{1}+w_{5}\right) u_{1}, \quad v_{1} B_{1}=\left(2 w_{1}+w_{5}\right) v_{1}, \\
& B_{2} u_{3}=\left(2 w_{3}+w_{5}\right) u_{3}, \quad v_{3} B_{2}=\left(2 w_{3}+w_{5}\right) v_{3}, \\
& B_{1} u_{3}+B_{2} u_{1}=2 w_{1} u_{3}+2 w_{3} u_{1}, \\
& v_{3} B_{1}+v_{1} B_{2}=2 w_{1} v_{3}+2 w_{3} v_{1}, \\
& \frac{1}{4}\left[B_{1}, B_{2}\right]=u_{1} v_{3}-u_{3} v_{1}, \\
& v_{1} u_{3}=v_{3} u_{1} .
\end{aligned}
$$

Since $2 w_{1}+w_{5}\left(\right.$ resp. $\left.2 w_{3}+w_{5}\right)$ is an eigenvalue of $B_{1}$ (resp. $B_{2}$ ), we have $2 w_{1}+w_{5} \in\left\{0, \alpha_{2}, \alpha_{3}\right\}$ and $2 w_{3}+w_{5} \in\left\{0, \beta_{2}, \beta_{3}\right\}$. First we consider the case $2 w_{1}+w_{5}=\alpha_{2}$ and $2 w_{3}+w_{5}=\beta_{2}$. We assume $a_{12} a_{23} \neq 0$, and then may take $a_{12}=a_{23}=1$. In this case, the eigenvectors are given by

$$
\begin{aligned}
& u_{1}=p_{1}\left(\begin{array}{c}
1 \\
\alpha_{2} \\
0
\end{array}\right), \quad v_{1}=q_{1}\left(\begin{array}{ll}
0 \alpha_{2}-\alpha_{3} & 1
\end{array}\right), \\
& u_{3}=p_{3}\left(\begin{array}{c}
0 \\
\beta_{2}-\beta_{3} \\
b_{32}
\end{array}\right), \quad v_{3}=q_{3}\left(b_{21} \beta_{2} 0\right) \text {, }
\end{aligned}
$$

where $p_{1}, q_{1}, p_{3}, q_{3} \in \mathbb{C}^{\times}$. Then the relations (3.16) can be regarded as a system of algebraic equations in $\alpha_{2}, \alpha_{3}, \beta_{2}, \beta_{3}, a_{13}, b_{21}, b_{31}, b_{32}, w_{5}, p_{1}, q_{1}, p_{3}$ and $q_{3}$. Solving them, we obtain

$$
\begin{aligned}
& 2 \alpha_{2}-\alpha_{3}=2 \beta_{2}-\beta_{3}, \\
& a_{13}=b_{31}=0, \\
& b_{32}=b_{21}+\alpha_{2} \beta_{2}-\left(\alpha_{2}-\alpha_{3}\right)\left(\beta_{2}-\beta_{3}\right), \\
& w_{5}=2 \alpha_{2}-\alpha_{3}, \\
& p_{3}=-p_{1}, \quad q_{1}=-\frac{1}{4 p_{1}}, \quad q_{3}=\frac{1}{4 p_{1}} .
\end{aligned}
$$

Then the entries of $B_{1}$ and $B_{2}$ are written in $\alpha_{2}, \alpha_{3}, \beta_{2}$ and $b_{21}$, and we have $B_{1}=A_{1}^{0}$ and $B_{2}=A_{2}^{0}$ if we set

$$
\begin{aligned}
& \alpha_{2}=a+c, \quad \alpha_{3}=2 a+2 c+g, \quad \beta_{2}=b+c, \\
& b_{21}=-c(2 a+2 b+2 c+g) .
\end{aligned}
$$

Thus in this case, the sets of the eigenvalues of $B_{1}, B_{2}$ and $-\left(B_{1}+B_{2}\right)$ are $\alpha^{0}, \beta^{0}$ and $\gamma^{0}$, respectively, and we have $\left(B_{1}, B_{2}\right) \in\left[\left(A_{1}^{0}, A_{2}^{0}\right)\right]$. Moreover we see that the Dotsenko-Fateev system (2.4) 
for generic parameters $(a, b, c, g)$ is obtained. It turns out that, if $a_{12} a_{23}=0$, any solution $\left(B_{1}, B_{2}\right)$ of (3.16) is reducible.

For the remaining eight cases, we have similar results. If $a_{12} a_{23} \neq 0$, we get irreducible representatives $\left(B_{1}, B_{2}\right)$ satisfying (3.9) for some $\rho_{1}, \rho_{2} \in \mathbb{C}$. If $a_{12} a_{23}=0$, any solution $\left(B_{1}, B_{2}\right)$ of (3.16) becomes reducible. This completes the proof.

Thus the prolongability uniquely determines a Fuchsian system of rank three with three singular points of spectral types $(1,1,1)$, which turns out to be the Dotsenko-Fateev system (2.4). This is another characterization of the Dotsenko-Fateev system.

On the other hand, the completely integrable system (3.7) is also determined uniquely. Namely, a restriction to a singular locus uniquely determines the completely integrable system.

Corollary 3.1. By the conditions given in Theorem 3.1, the completely integrable system (3.7) is uniquely determined by using the parameters $(a, b, c, g)$ up to gauge transformations by $\mathrm{GL}(4, \mathbb{C})$ and the transformation $V(x, y) \mapsto x^{\lambda_{1}} y^{\lambda_{2}}(x-1)^{\lambda_{3}}(y-1)^{\lambda_{4}} V(x, y)$.

Proof. We have only to check that, in the proof of Theorem 3.1, the entries of $R_{1}, R_{2}, R_{3}$ and $R_{4}$ are uniquely determined. After preliminary gauge transformations, we see that $S_{j}(1 \leq j \leq 4)$ are given by (3.14) in terms of $B_{1}, B_{2}$. We also have (3.15), $2 w_{1}+w_{5} \in\left\{0, \alpha_{2}, \alpha_{3}\right\}$ and $2 w_{3}+w_{5} \in\left\{0, \beta_{2}, \beta_{3}\right\}$. We consider the case $2 w_{1}+w_{5}=\alpha_{2}, 2 w_{3}+w_{5}=\beta_{2}$, so that we get (3.17) and (3.18). Then, by setting (3.19), $B_{1}, B_{2}, w_{j}(1 \leq j \leq 5)$ are written by using the parameters $(a, b, c, g)$. The remaining $u_{j}, v_{j}(1 \leq j \leq 4)$ are determined by (3.12) and (3.17), where $p_{1}$ is the only undetermined parameter. We can delete $p_{1}$ by the gauge transformation by $\operatorname{diag}\left[1,1,1, p_{1}\right]$. The other cases are similar.

\section{Middle Convolution}

The middle convolution is introduced by Katz [7] as an operation for local systems, and then defined for Fuchsian systems of ordinary differential equations by Dettweiler and Reiter [1]. It is a fundamental operation for Fuchsian systems, which leaves the number of accessory parameters and the deformation equation invariant ( [7], [1], [5]). We like to consider a relation between the middle convolution and prolongability.

It seems natural to ask whether a middle convolution image of a prolongable Fuchsian system is also prolongable. Oshima remarked that the middle convolution image of the Dotsenko-Fateev system (2.4) coincides with a restriction equation of Heckman-Opdam hypergeometric system of $B C_{2}$ type to some singular locus (cf. [8]). Thus the prolongability is preserved by the middle convolution in this case. We have another natural question. For completely integrable systems, can we define a middle convolution which is compatible with prolongations and restrictions? To study this question, we define a middle convolution for completely integrable systems of KZ type. In [4] we generalized the definition for completely integrable systems with logarithmic singularities along hyperplane arrangements, and studied basic properties.

Let $a_{1}, a_{2}, \ldots, a_{p}$ be distinct points in $\mathbb{C}$, and $n$ an integer. A completely integrable systems of $\mathrm{KZ}$ type is a Pfaffian system of the form

$$
d U=\left[\sum_{i=1}^{q} \sum_{j=1}^{p} A_{i j} \frac{d x_{i}}{x_{i}-a_{j}}+\sum_{1 \leq i<i^{\prime} \leq q} B_{i i^{\prime}} \frac{d\left(x_{i}-x_{i^{\prime}}\right)}{x_{i}-x_{i^{\prime}}}\right] U,
$$

where $A_{i j}, B_{i i^{\prime}} \in \mathfrak{g l}(n, \mathbb{C})$. For $i>i^{\prime}$ we set $B_{i i^{\prime}}=B_{i^{\prime} i}$. 
Take a $\mu \in \mathbb{C}$. For $1 \leq k \leq q$, we define a convolution of (4.1) in $x_{k}$-direction with parameter $\mu$ by the Pfaffian system satisfied by

$$
\hat{U}={ }^{t}\left(V_{1}, \ldots, V_{p}, W_{1}, \ldots, W_{k-1}, W_{k+1}, \ldots, W_{q}\right),
$$

where

$$
V_{j}\left(x_{1}, \ldots, x_{q}\right)=\int_{\Delta} \frac{U\left(x_{1}, \ldots, x_{k-1}, t, x_{k+1}, \ldots, x_{q}\right)}{t-a_{j}}\left(t-x_{k}\right)^{\mu} d t
$$

for $1 \leq j \leq p$,

$$
W_{i}\left(x_{1}, \ldots, x_{q}\right)=\int_{\Delta} \frac{U\left(x_{1}, \ldots, x_{k-1}, t, x_{k+1}, \ldots, x_{q}\right)}{t-x_{i}}\left(t-x_{k}\right)^{\mu} d t
$$

for $1 \leq i \leq q, i \neq k$, and $U\left(x_{1}, \ldots, x_{q}\right)$ is a solution of (4.1). For simplicity we take $k=q$, and consider a convolution in $x_{q}$-direction.

The partial differential equation for $\hat{U}$ with respect to $x_{q}$ is nothing but the convolution as an ordinary differential equation of the partial differential equation of $U$ with respect to $x_{q}$, and is given by

$$
\frac{\partial \hat{U}}{\partial x_{q}}=\left(\sum_{j=1}^{p} \frac{G_{q j}}{x_{q}-a_{j}}+\sum_{i=1}^{q-1} \frac{H_{q i}}{x_{q}-x_{i}}\right) \hat{U},
$$

where $G_{q j}$ and $H_{q i}$ are $(p+q-1) n \times(p+q-1) n$-matrices defined by

$$
G_{q j}=\sum_{k=1}^{p} E_{j k} \otimes A_{q k}+\sum_{l=1}^{q-1} E_{j p+l} \otimes B_{q l}+E_{j j} \otimes \mu I_{n}
$$

for $1 \leq j \leq p$ and

$$
H_{q i}=\sum_{k=1}^{p} E_{p+i k} \otimes A_{q k}+\sum_{l=1}^{q-1} E_{p+i p+l} \otimes B_{q l}+E_{p+i p+i} \otimes \mu I_{n}
$$

for $1 \leq i \leq q-1$ ( [2]). Here $E_{i j}$ denotes the $(p+q-1) \times(p+q-1)$-matrix with the only non-zero entry 1 at $(i, j)$-position.

The partial derivatives of $V_{j}$ and $W_{i}$ with respect to $x_{k}(1 \leq k \leq q-1)$ are calculated in a straightforward way. Thus we obtain the Pfaffian system

$$
d \hat{U}=\left[\sum_{i=1}^{q} \sum_{j=1}^{p} G_{i j} \frac{d x_{i}}{x_{i}-a_{j}}+\sum_{1 \leq i<i^{\prime} \leq q} H_{i i^{\prime}} \frac{d\left(x_{i}-x_{i^{\prime}}\right)}{x_{i}-x_{i^{\prime}}}\right] \hat{U}
$$

for $\hat{U}$, where

$$
\begin{aligned}
G_{k j}= & \sum_{l=1}^{p+q-1} E_{l l} \otimes A_{k j}+E_{j j} \otimes B_{k q}-E_{j p+k} \otimes B_{k q}-E_{p+k j} \otimes A_{q j} \\
& +E_{p+k p+k} \otimes A_{q j} \quad(1 \leq j \leq p), \\
H_{k i}= & \sum_{l=1}^{p+q-1} E_{l l} \otimes B_{k i}+E_{p+i p+i} \otimes B_{k q}-E_{p+i p+k} \otimes B_{k q}-E_{p+k p+i} \otimes B_{q i} \\
& +E_{p+k p+k} \otimes B_{q i} \quad(1 \leq i \leq q-1, i \neq k), \\
H_{k q}= & H_{q k} .
\end{aligned}
$$


This Pfaffian system is completely integrable if (4.1) is so.

For the Pfaffian system (4.1) and the parameter $\mu$, we define the subspaces $\mathscr{K}$ and $\mathscr{L}$ of $\mathbb{C}^{(p+q-1) n}$ by

$$
\begin{aligned}
& \mathscr{K}=\left\{\left(\begin{array}{c}
v_{1} \\
\vdots \\
v_{p} \\
w_{1} \\
\vdots \\
w_{q-1}
\end{array}\right) ; v_{j} \in \operatorname{Ker} A_{q j}(1 \leq j \leq p), w_{i} \in \operatorname{Ker} B_{q i}(1 \leq i \leq q-1)\right\}, \\
& \mathscr{L}=\operatorname{Ker}\left(G_{q 1}+\cdots+G_{q p}+H_{q 1}+\cdots+H_{q q-1}\right) .
\end{aligned}
$$

Then we have the following.

Lemma 4.1. For $1 \leq i, i^{\prime} \leq q, 1 \leq j \leq p$ with $i \neq i^{\prime}, \mathscr{K}$ and $\mathscr{L}$ are invariant subspaces of $G_{i j}$ and $H_{i i^{\prime}}$.

Definition 4.1. For $1 \leq i, i^{\prime} \leq q, 1 \leq j \leq p$ with $i \neq i^{\prime}$, let $\bar{G}_{i j}$ (resp. $\bar{H}_{i i^{\prime}}$ ) be the action of $G_{i j}$ (resp. $\left.H_{i i^{\prime}}\right)$ on the quotient space $\mathbb{C}^{(p+q-1) n} /(\mathscr{K}+\mathscr{L})$. The Pfaffian system

$$
d \bar{U}=\left[\sum_{i=1}^{q} \sum_{j=1}^{p} \bar{G}_{i j} \frac{d x_{i}}{x_{i}-a_{j}}+\sum_{1 \leq i<i^{\prime} \leq q} \bar{H}_{i i^{\prime}} \frac{d\left(x_{i}-x_{i^{\prime}}\right)}{x_{i}-x_{i^{\prime}}}\right] \bar{U}
$$

is called the middle convolution of the system (4.1) in $x_{q}$-direction with parameter $\mu$. We denote by $m c_{\mu}^{x_{q}}$ the operation to give the middle convolution in $x_{q}$-direction with parameter $\mu$.

The middle convolution in $x_{k}$-direction with parameter $\mu$ for $1 \leq k \leq q-1$ can also be defined in a similar way. If we consider the one-dimensional section in $x_{k}$-direction, the middle convolution in $x_{k}$-direction is just the middle convolution for the system of ordinary differential equations. Then, thanks to [7], [1] and [2], if the one-dimensional section of (4.1) in $x_{k}$-direction is irreducible, the middle convolution of (4.1) in $x_{k}$-direction with parameter $\mu$ is completely integrable and irreducible. Moreover we have the additivity

$$
\begin{aligned}
m c_{0}^{x_{k}} & =\mathrm{id}, \\
m c_{\mu_{1}}^{x_{k}} m c_{\mu_{2}}^{x_{k}} & =m c_{\mu_{1}+\mu_{2}}^{x_{k}} .
\end{aligned}
$$

Now we apply the middle convolution to the Pfaffian system studied in Theorem 3.1. By Corollary 3.1, the Pfaffian system is essentially (2.5) with (2.6), so that we consider the middle convolution of (2.5). First we consider the middle convolution in $x$-direction. By (2.6) we see that $\operatorname{dim} \operatorname{Ker} P_{1}=\operatorname{dim} \operatorname{Ker} P_{3}=2$ and $\operatorname{dim} \operatorname{Ker} P_{5}=3$. Hence we get $\operatorname{dim} \mathscr{K}=2+2+3=7$. If we take $\mu \neq 0, \alpha, \beta$ as a parameter of the middle convolution, we have $\mathscr{L}=\{0\}$. Then in this case the rank of the middle convolution becomes $3 \times 4-7=5$, and we get the system

$$
d \bar{V}=\left[\bar{P}_{1} \frac{d x}{x}+\bar{P}_{2} \frac{d y}{y}+\bar{P}_{3} \frac{d x}{x-1}+\bar{P}_{4} \frac{d y}{y-1}+\bar{P}_{5} \frac{d(x-y)}{x-y}\right] \bar{V}
$$


with $\bar{P}_{j} \in \mathfrak{g l}(5, \mathbb{C})$. We see that $\operatorname{dim} \operatorname{Ker} \bar{P}_{5}=4$, and hence the restriction of (4.2) to the singular locus $x=y$ becomes a system

$$
\frac{d U}{d x}=\left(\frac{S_{1}}{x}+\frac{S_{2}}{x-1}\right) U
$$

of rank four. Explicitly we have

$$
\begin{aligned}
& S_{1}=\left(\begin{array}{cccc}
\mu+1-c+\varepsilon & \frac{\varepsilon}{1-\gamma-\varepsilon} & -\frac{1}{1-\gamma-\varepsilon} & 0 \\
0 & 1-\gamma & 0 & 0 \\
0 & \alpha \beta+\gamma^{\prime} \varepsilon & 0 & 0 \\
0 & -(\alpha+1-\gamma) & 0 & \mu+2(1-\gamma)
\end{array}\right), \\
& S_{2}= \\
& \left(\begin{array}{cccc}
-\gamma^{\prime}-\varepsilon & -\frac{\varepsilon}{1-\gamma-\varepsilon} & \frac{1}{1-\gamma-\varepsilon} & 0 \\
-(\mu+2 \varepsilon) \varepsilon & \mu+2\left(\varepsilon-\gamma^{\prime}\right)-\frac{(1-\gamma) \varepsilon}{1-\gamma-\varepsilon} & -\frac{1-\gamma-2 \varepsilon}{1-\gamma-\varepsilon} & -\frac{(\beta+1-\gamma)(1-\gamma)}{1-\gamma-\varepsilon} \\
-(\mu+2 \varepsilon)\left(\alpha \beta+(1-\gamma) \gamma^{\prime}\right) & -\frac{\varepsilon\left(\alpha \beta+\gamma^{\prime}(2-2 \gamma-\varepsilon)\right.}{1-\gamma-\varepsilon} & \mu+\frac{\alpha \beta+\gamma^{\prime} \varepsilon}{1-\gamma-\varepsilon} & \frac{(\beta+1-\gamma)\left(\alpha \beta+\gamma^{\prime} \varepsilon\right)}{1-\gamma-\varepsilon} \\
(\mu+2 \varepsilon)(\alpha+1-\gamma) & \frac{(\alpha+1-\gamma) \varepsilon}{1-\gamma-\varepsilon} & -\frac{\alpha+1-\gamma}{1-\gamma-\varepsilon} & \frac{(\alpha+1-\gamma)(\beta+1-\gamma)}{1-\gamma-\varepsilon}
\end{array}\right) .
\end{aligned}
$$

We see that the eigenvalues of $S_{1}, S_{2}$ and $-\left(S_{1}+S_{2}\right)$ are as in the following table, and these matrices are diagonalizable if $\mu$ is generic.

\begin{tabular}{|c|c|}
\hline matrix & eigenvalues \\
\hline$S_{1}$ & $0,1-\gamma, \mu+2-2 \gamma, \mu-\alpha-\beta+\gamma^{\prime}$ \\
\hline$S_{2}$ & $0,-\gamma^{\prime}, \mu-1-\alpha-\beta+\gamma, \mu-2 \gamma^{\prime}$ \\
\hline$-\left(S_{1}+S_{2}\right)$ & $2 \alpha-\mu, 2 \beta-\mu, \gamma+\gamma^{\prime}-1-\mu, \gamma+\gamma^{\prime}-1-\mu$ \\
\hline
\end{tabular}

Thus, if $\mu$ is generic, the spectral types of $S_{1}, S_{2}$ and $-\left(S_{1}+S_{2}\right)$ are $(1,1,1,1),(1,1,1,1)$ and $(2,1,1)$, respectively, and then the index of rigidity of the system (4.3) is -2 .

There are several choices of $\mu$ which change the spectral type of $S_{1}, S_{2}$ or $-\left(S_{1}+S_{2}\right)$ while keeping the condition $\mu \neq 0, \alpha, \beta$. If, for example, we choose $\mu=\gamma-1$, the multiplicity of the eigenvalue $1-\gamma$ of $S_{1}$ becomes two, while the dimention of the eigenspace is still one, and hence the index of rigidity is unchanged. By excluding such cases, there remain two choices $\mu=2 \gamma-2$ and $\mu=2 \gamma^{\prime}$ which change the index of rigidity to zero. Since these choices give equivalent systems, we take $\mu=2 \gamma-2$. By a fractional linear transformation of the independent variable and a gauge transformation, we can send the system (4.3) with $\mu=2 \gamma-2$ to the system

$$
\frac{d U}{d x}=\left(\frac{T_{1}}{x}+\frac{T_{2}}{x-1}\right) U
$$

where

$$
\begin{aligned}
& T_{1}=S_{1}, \\
& T_{2}=\left(\gamma-\gamma^{\prime}-1\right) I-\left(S_{1}+S_{2}\right) .
\end{aligned}
$$

The eigenvalues are given by the following, and the dimensions of the 0 -eingenspaces of $T_{1}$ and $T_{2}$ are two. 


\begin{tabular}{|c|c|}
\hline matrix & eigenvalues \\
\hline$T_{1}$ & $0,0,1-\gamma,-\alpha-\beta+2 \gamma+\gamma^{\prime}-2$ \\
\hline$T_{2}$ & $0,0,2 \alpha-\gamma-\gamma^{\prime}+1,2 \beta-\gamma-\gamma^{\prime}-1$ \\
\hline$-\left(T_{1}+T_{2}\right)$ & $\gamma-\gamma^{\prime}-1,-\gamma+\gamma^{\prime}+1, \gamma-1, \alpha+\beta-2 \gamma-\gamma^{\prime}+2$ \\
\hline
\end{tabular}

Proposition 4.1. The system (4.4) is connected with the Dotsenko-Fateev system (2.4) by a middle convolution and an addition.

Proof. By a middle convolution with parameter $\alpha$, the system (4.4) is sent to a system

$$
\frac{d \bar{U}}{d x}=\left(\frac{\bar{T}_{1}}{x}+\frac{\bar{T}_{2}}{x-1}\right) \bar{U}
$$

of rank three. Then operating the addition

$$
\left(\bar{T}_{1}, \bar{T}_{2}\right) \mapsto\left(\bar{T}_{1}, \bar{T}_{2}+\left(\alpha+2 \beta+\gamma^{\prime}\right) I\right)
$$

we get the Dotsenko-Fateev system (2.4) with parameter $(a, b, c, g)$, where

$$
\begin{aligned}
& a=\frac{\gamma}{2}-\frac{\gamma^{\prime}}{2}, b=\beta+\frac{\gamma}{2}+\frac{\gamma^{\prime}}{2}, \\
& c=\alpha+\beta-\frac{\gamma}{2}+\frac{\gamma^{\prime}}{2}, g=-2 \beta .
\end{aligned}
$$

By noting that the inverses of an addition and a middle convolution are given by an addition and a middle convolution, respectively, the above result tells that the following diagram is commutative if we take the special value $\mu=2 \gamma-2$ or $\mu=2 \gamma^{\prime}$ of the middle convolution parameter:

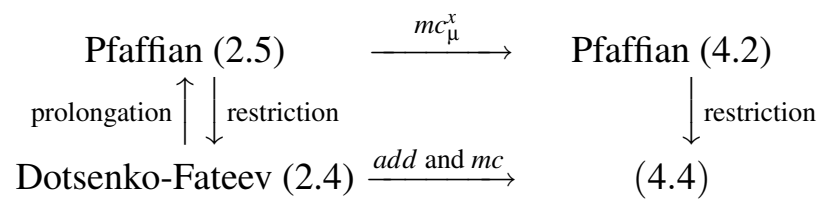

For other values of $\mu,(2.4)$ is not connected to the restriction of (4.2) by additions and middle convolutions, because the indexes of rigidity are different. This may be an interesting feature of the middle convolution of Pfaffian systems.

The middle convolution of the same Pfaffian system (2.5) in $y$-direction gives an essentially same system

$$
d \bar{V}=\left[\bar{P}_{2} \frac{d x}{x}+\bar{P}_{1} \frac{d y}{y}+\bar{P}_{4} \frac{d x}{x-1}+\bar{P}_{3} \frac{d y}{y-1}+\bar{P}_{5} \frac{d(x-y)}{x-y}\right] \bar{V}
$$

where $\bar{P}_{1}, \bar{P}_{2}, \ldots, \bar{P}_{5}$ are the same ones in the system (4.2). This is obtained from (4.2) by exchanging $x$ and $y$.

\section{Acknowledgments}

This work is supported by the JSPS grant-in-aid for scientific research B, No.21340038. 


\section{References}

[1] M. Dettweiler and S. Reiter, An algorithm of Katz and its application to the inverse Galois problem, $J$. Symbolic Comput. 30 (2000), 761-798.

[2] - Middle convolution of Fuchsian systems and the construction of rigid differential systems, J. Algebra 318 (2007), 1-24.

[3] V. S. Dotsenko and V. A. Fateev, Conformal algebra and multipoint correlation function in 2D statistical models, Nuclear Phys. B 240 (1984), 312-348.

[4] Y. Haraoka, Middle convolution for completely integrable systems with logarithmic singularities along hyperplane arrangements, Adv. Stud. Pure Math. 62 (2012), 109-136.

[5] Y. Haraoka and G. Filipuk, Middle convolution and deformation for Fuchsian systems, J. London Math. Soc. 76 (2007), 438-450.

[6] M. Kato, Connection formulas for Appells system $F_{4}$ and some applications, Funkcial. Ekvac. 38 (1995), 243-266.

[7] N. M. Katz, Rigid Local Systems, (Princeton Univ. Press, Princeton, NJ, 1996).

[8] T. Oshima and N. Shimeno, Heckman-Opdam hypergeometric functions and their specializations, RIMS Kôkyûroku Bessatsu B20 (2010), 129-162. 